\title{
A lump in an arm
}

\author{
Federica Pederiva $\cdot$ Edoardo Guida • \\ Giulio Andrea Zanazzo • Massimo Gregori • \\ Jurgen Schleef
}

Received: 11 July 2013 / Accepted: 18 July 2013 / Published online: 6 August 2013

(C) Springer-Verlag Berlin Heidelberg 2013

\begin{abstract}
The authors present a magnetic resonance image showing a soft tissue mass misdiagnosed as sarcoma.
\end{abstract}

Keywords Nodular fasciitis · Soft tissue mass

A 12-year-old boy presented with a painless, rapidly growing swelling mass in his right arm without any other accompanying symptoms or history of trauma. A $4 \times 2-\mathrm{cm}$ solid fixed mass with normal overlying skin was palpated. Laboratory tests and tumor markers were unremarkable.

Magnetic resonance imaging showed an enhancing mass inside the triceps brachii muscle reached by two vessels (Fig. 1). Excisional biopsy was performed and histopathology revealed nodular fasciitis.

Nodular fasciitis is a benign reactive fibroblastic and myofibroblastic proliferation most commonly diagnosed in adults. Ten percent of the lesions occur in children especially in the extremities or trunk and also in the head and neck. It is usually subcutaneous in distribution but sometimes also in intramuscular and intermuscular locations involving skeletal muscle. The etiology is still unknown and whether the reactive process is triggered by local injury or inflammatory processes is not resolved. It often develops rapidly, which may be concerning for malignancy, being the most common benign mesenchymal lesion misdiagnosed as sarcoma. Spontaneous regression is the natural history and a proper diagnosis is essential to avoid unnecessarily aggressive treatment [1-3]. Although relatively rare, nodular fasciitis should be considered in the differential diagnosis in any pediatric patient with a soft tissue mass.

F. Pederiva $(\bowtie) \cdot$ E. Guida $\cdot$ G. A. Zanazzo $\cdot$ M. Gregori $\cdot$ J. Schleef Institute for Maternal and Child Health - IRCCS "Burlo Garofolo", Via dell'Istria 65/1, 34137 Trieste, Italy

e-mail: federica_pederiva@yahoo.it

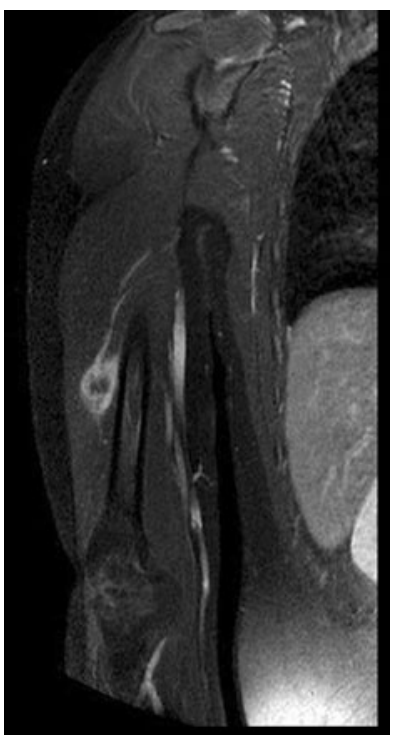

Fig. 1 Axial enhanced MRI of the right arm showing a hyperintense well-circumscribed mass inside the triceps brachii muscle. Two vessels reach the nodule

Conflict of interest There is no financial assistance or any potential conflict of interest, real or perceived.

\section{References}

1. Hutter RV, Stewart FW, Foote FW Jr (1962) Fasciitis. A report of 70 cases with follow-up proving the benignity of the lesion. Cancer 15:992-1003

2. Tomita S, Thompson K, Carver T, Vazquez WD (2009) Nodular fasciitis: a sarcomatous impersonator. J Pediatr Surg 44:e17-e19

3. Weinreb I, Shaw AJ, Perez-Ordonez B, Goldblum JR, Rubin BP (2009) Nodular fasciitis of the head and neck region: a clinicopathologic description in a series of 30 cases. J Cutan Pathol 36:1168-1173 